\title{
Vol. 69, No. 49
}

In the report "COVID-19 Mortality Among American Indian and Alaska Native Persons - 14 States, JanuaryJune 2020," on page 1853, the list of authors should have read "Jessica Arrazola, DrPH"; Matthew M. Masiello'; Sujata Joshi, $\mathrm{MSPH}^{2}$; Adrian E. Dominguez, MS³; Amy Poel, $\mathrm{MPH}^{3}$; Crisandra M. Wilkie, $\mathrm{MPH}^{3}$; Jonathan M. Bressler, $\mathrm{MPH}^{4}$; Joseph McLaughlin, $\mathrm{MD}^{4}$; Jennifer Kraszewski, MS5; Kenneth K. Komatsu, $\mathrm{MPH}^{6}$; Xandy Peterson Pompa, $\mathrm{MPH}^{6}$; Megan Jespersen, $\mathrm{MPH}^{7}$; Gillian Richardson, $\mathrm{MPH}^{7}$; Nicholas Lehnertz, $\mathrm{MD}^{8}$; Pamela LeMaster, $\mathrm{PhD}^{8}$; Britney Rust, $\mathrm{MPH}^{9}$; Alison Keyser Metobo, $\mathrm{MPH}^{10}$; Brooke Doman, $\mathrm{MPH}^{11}$; David Casey, $\mathrm{MA}^{12}$; Jessica Kumar, DO ${ }^{12}$; Alyssa L. Rowell, MA ${ }^{12}$; Tracy K. Miller, PhD ${ }^{13}$; Mike Mannell, MPH ${ }^{14}$; Ozair Naqvi, MS ${ }^{14}$; Aaron M. Wendelboe, $\mathrm{PhD}^{14}$; Richard Leman, $\mathrm{MD}^{15}$; Joshua L. Clayton, $\mathrm{PhD}^{16}$; Bree Barbeau, $\mathrm{MPH}^{17}$; Samantha K. Rice, $\mathrm{MPH}^{18}$; Samantha J.H. Rolland, $\mathbf{P h D}^{\mathbf{1 8}}$; Victoria Warren-Mears, $\mathrm{PhD}^{2}$; Abigail Echo-Hawk, MA³; Andria Apostolou, $\mathrm{PhD}^{19}$; Michael Landen, $\mathrm{MD}^{1}$.

The affiliation for Samantha J.H. Rolland is Washington State Department of Health. 